\title{
DIFICULTADES DE APRENDIZAJE EN QUÍMICA: CARACTERIZACIÓN Y BÚSQUEDA DE ALTERNATIVAS PARA SUPERARLAS
}

\author{
Learning difficulties in Chemistry: characterization \\ and search for alternatives to overcome them
}

Fidel Antonio Cárdenas S. ${ }^{1}$

Resumen: Es generalmente aceptado que muchos estudiantes encuentran difícil de aprender unos temas de Química más que otros, siendo los mas frecuentes el de soluciones, estequiometría, la ecuación de estado, el equilibrio químico y las soluciones buffer. Por lo menos en parte, estas dificultades pueden explicarse teniendo en cuenta factores internos al los estudiantes como su capacidad de procesamiento de información y factores externos como la naturaleza propia de la Química. En una investigación llevada a cabo con estudiantes de un grupo de Química General en la Universidad de La Salle, se encontró que los estudiantes presentan dificultades de aprendizaje con estos mismos temas y que a pesar de que la mayoría de ellos presentó altos valores de capacidad mental, sus resultados no fueron tan buenos como lo predice la teoría pero mostraron tendencia hacia ellos. En aquellos estudiantes que mostraron los valores mas bajos de capacidad mental, sus desempeños también fueron bajos; sin embargo, se pudo establecer que las preguntas formuladas para las evaluaciones, y en particular para el examen final, tenían una alta demanda y se relacionaban con los temas que los alumnos consideran de mayor dificultad; la confluencia de estos factores se propone como explicación posible para el grado de aprendizaje de este grupo.

Palavras-chave: ensino de Química. desempenho acadêmico. processo de avaliação.

\begin{abstract}
It is generally accepted that many students have learning difficulties with topics such as stequiometry, solutions, chemical equilibrium, buffer solutions and gaseous states. Some of the learning difficulties in chemistry can be accounted for in terms of the learner's mental capacity to process information and the nature of chemistry as science. An investigation carried out involving general chemistry students in the University of La Salle, led to the conclusion that although many of the participants had high mental capacity values their academic performance was low. Academic performance for those students with low mental capacity values was also low. A possible explanation for these results may stem from the fact that many questions included in the assessment process were found to be of high demand and were related to chemistry topics considered by the students as the most difficult in general Chemistry.
\end{abstract}

Keywords: Chemistry education. academic performance. assessment process.

\footnotetext{
${ }^{1}$ Docente investigador, División de Formación Avanzada, Universidad de La Salle, Bogotá, Colômbia. E-mail:fcardenas@lasalle.edu.co; fidelantonio@gmail.com
} 


\section{Introducción}

Es un hecho conocido, que muchos de los estudiantes durante la educación secundaria e incluso en la Universidad, al enfrentarse en su carrera, al estudio de la Química, unos más que otros, encuentran dificultades de aprendizaje en general y en particular para ciertos temas de esta ciencia. Tales dificultades se manifiestan principalmente en bajo rendimiento académico, poco interés por su estudio, repitencia y usualmente una actitud pasiva en el aula. ¿Por qué los estudiante tienen dificultades para aprender algunos temas más que otros?, ¿Qué relación existe entre las dificultades de aprendizaje, la desmotivación y la pasividad de los alumnos en las clases de Química?, son algunas de las preguntas que en este contexto ameritan la búsqueda sistemática de respuestas.

La investigación en el campo de las dificultades de aprendizaje en el mundo, ha logrado caracterizar y establecer una serie de temas de Química acerca de los cuales la mayoría de los estudiantes que se encuentran por primera vez con ellos, e incluso muchos de los docentes, tienen dificultades para su aprendizaje, es el caso de las soluciones sobresaturadas, el equilibrio químico, las reacciones de oxidación reducción, las velocidades de reacción, la estequiometría y la ecuación de estado entre otros; ¿tienen los estudiantes de la Universidad de La Salle dificultades con estos mismos temas?, ¿cuáles son los tópicos más frecuentes que presentan dificultades para la mayoría de los estudiantes?, ¿qué estrategias han desarrollado los alumnos para enfrentar con éxito estos y otros temas de Química?, son otras preguntas que se adicionan a las ya mencionadas.

Es posible que muchas de estas dificultades tengan origen interno, otras origen externo al estudiante, o quizá se presente una combinación de los dos tipos; puesto que muchas de las dificultades están mas allá de una posible acción de los docentes e incluso de la Universidad, como es el caso de aquellas de origen genético y de algunas de origen económico y social. El estudio y la caracterización de las dificultades de aprendizaje a que se refiere este artículo se circunscriben a las de origen interno que sean susceptibles de establecer y caracterizar, como la capacidad mental de los alumnos y sus relaciones con la demanda de una tarea, las derivadas de la preparación académica previa de los alumnos, y las derivadas de la naturaleza propia de la Química.

Los resultados que se presentan a continuación provienen de una investigación que se adelantó en la Universidad de La Salle, acerca de las dificultades de aprendizaje en Química que se presentan en los estudiantes de los primeros semestres; específicamente se procuraba establecer los tópicos de mayor dificultad para los estudiantes y proponer explicaciones internas y externas para las dificultades de aprendizaje asociadas a los mismos.

\section{Marco de referencia}

En procura de hacer más funcional la teoría de Piaget, Pascual-Leone en 1969 formuló la teoría neo-Piagetiana, la cual ha sido modificada y ampliada desde aquel tiempo, (PascualLeone, 1970). La teoría postula que el mecanismo central de atención o memoria de trabajo, operador M, es altamente responsable por el progreso del desarrollo del niño a través de los estados Piagetianos y hace énfasis en las relaciones existentes entre este parámetro M y la demanda de la tarea Z. 
Dificultades de aprendizaje en Química...

Los planteamientos originales de Pascual-Leone (1970) han sido ampliados y modificados, por él mismo y sus colaboradores, particularmente en el sentido de determinar cuantitativamente el parámetro M, para lo cual se emplea del Test de las Figuras de Intersección, FIT, y el Test de los Dígitos Invertidos, DBT (Case, 1972; Case, 1974; Case e Globerson, 1974; Scardamalia, 1977).

Haciendo uso de los planteamientos de Pascual-Leone (1970), es posible buscar explicaciones para las dificultades que presentan algunos estudiantes en la adquisición y dominio de nuevas habilidades y conocimientos, en término de: la aplicación de una estrategia razonable pero muy simplificada, una sobre carga de instrucciones en la memoria de trabajo o una insuficiente familiarización con las operaciones básicas requeridas para resolver el problema. Si éste es el caso, los correctivos pedagógicos tendientes a eliminar estas dificultades serían: diagnosticar la estructura incorrecta, mostrar a los alumnos por qué es inadecuada y orientarlos para formular otra mejor; disminuir la carga de la memoria de trabajo minimizando la información y aumentar la práctica de las operaciones básicas, entre las cuales se encuentran aquellas de procesamiento y agrupación de información. Estas operaciones básicas dependen del campo de conocimiento al cual pertenezca una tarea, y, por lo tanto, en el caso de esta investigación, varían con el contexto conceptual de la Química, dentro del cual se han formulado las preguntas. Los procedimientos anteriores parecen aplicables a la resolución de cualquier tarea, independientemente de que ésta se refiera a desarrollo cognitivo, literatura o a cualquier tópico de las asignaturas convencionales.

A mediados de los ochenta, Alex H. Johnstone y uno de sus colaboradores (Johnstone e El-Banna, 1986) publicaron un artículo en el cual presentaban un modelo que tuvo sus orígenes en otros trabajos que realizó conjuntamente con Kellet y Wham (Johnstone e Kellet, 1980; Johnstone e Wham, 1982; Johnstone, 1984; Cassels e Johnstone, 1984), a finales de la década del 70 y comienzos del 80. Este modelo se describe e ilustra a continuación:

De una manera simplificada, el modelo se presenta en la Figura 1. En esta figura, el espacio M aparece compuesto por dos áreas X y Y, X representa el espacio donde un ser humano mantiene y procesa información y, Y, representa el espacio de las estrategias, "trucos" y técnicas que el hombre utiliza para procesar dicha información.

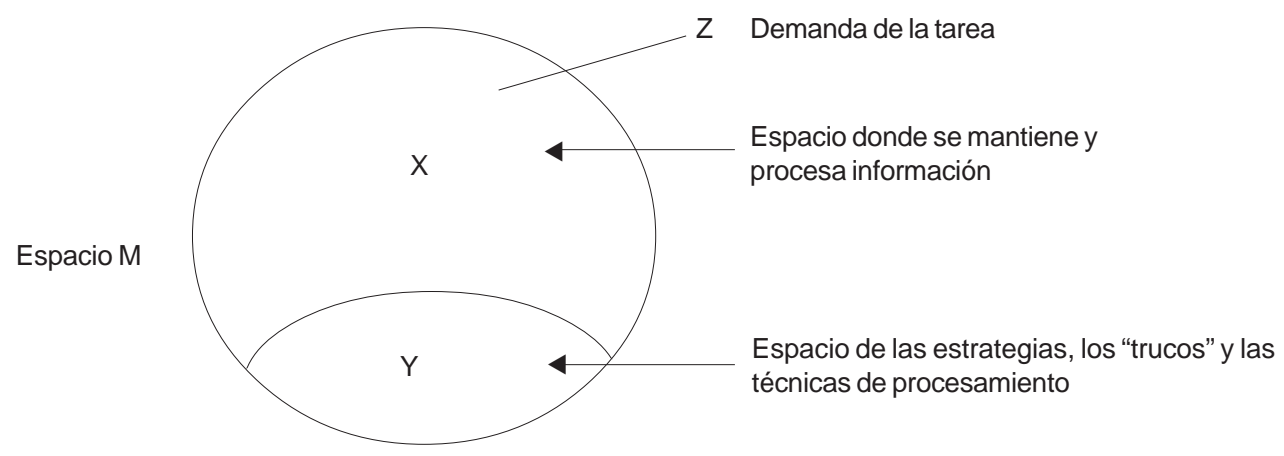

Figura 1. Modelo propuesto por Johnstone e El-Banna para el espacio M (Johnstone e El-Banna, 1986). 
Contrario a lo que puede apreciarse a simple vista en la Figura 1, es pertinente aclarar que el espacio M no está compuesto por dos sistemas diferentes. El modelo en realidad significa que para un individuo adulto el parámetro $\mathrm{M}$, que además es constante, es el espacio total de pensamiento y retención de información, donde él es capaz, no solamente de retenerla, sino también de pensar y actuar mentalmente sobre ella, conjuntamente con el espacio ocupado por el plano funcional Y, sobre el cual puede coordinar los ítems de información. De esta manera, el espacio M consta de la suma conceptual de los componentes X y Y.

El componente $\mathrm{X}$ hace referencia al número máximo de ítems informativos que un estudiante puede tener en su mente cuando desarrolla una tarea, mientras que el componente Y representa la estrategia funcional requerida para organizar y procesar la información en conjunto.

Con dicho modelo se ha pretendido explicar el éxito y/o el fracaso de muchos estudiantes en su proceso de aprendizaje, de aspectos referentes básicamente a la Química, teniendo siempre en cuenta las limitaciones asociadas con la medición del parámetro M o capacidad mental de los alumnos (Al-Naeme, 1989).

\section{Los instrumentos de recolección de datos}

Desde el punto de vista teórico, los instrumentos empleados para establecer la capacidad mental de un individuo o grupo de individuos, deben tener las siguientes dos características: exigir de parte del sujeto retener o memorizar información transitoriamente y, operar mentalmente sobre ella, es decir procesar esa información. El Test de las Figuras de Intersección, FIT, y el Test de los Dígitos Invertidos, DBT, que se emplean en esta investigación, cumplen estos dos requerimientos y por eso se usan para tal propósito. Es de anotar, que estos instrumentos, se consideran instrumentos estandarizados ampliamente, aplicados en el área de investigación del Aprendizaje en Química (Johnstone e El-Banna, 1986; El-Banna, 1987; PascualLeone, 1967).

Además de los instrumentos anteriores, se diseñó y elaboró específicamente un cuestionario para recoger información personal de los participantes, su trayectoria académica, tiempo dedicado al estudio de la Química, temas de mayor dificultad encontrados en el curso y posibles causas a las cuales aducen sus dificultades de aprendizaje.

Así mismo, se constituyeron en instrumentos de recolección de datos las evaluaciones y los textos de los exámenes utilizados por los docentes en el desarrollo del curso, en cuanto fueron la base para establecer la demanda de las tareas que posteriormente se contrastaron con la capacidad mental de sus autores, los estudiantes que conformaron el curso.

\section{El proceso para establecer la demanda de una pregunta}

La demanda de una tarea o de una pregunta, corresponde al mayor número de pasos o de esquemas de pensamiento que debe emplear el estudiante menos eficiente de una clase para resolverla con excito. La demanda de una tarea es diferente para cada persona, varía con los conocimientos previos de los estudiantes, su capacidad para organizar y procesar información 
Dificultades de aprendizaje en Química...

y, con las condiciones donde se lleva acabo el aprendizaje. Así, la demanda de una tarea representa el esfuerzo mental, en términos de procesamiento de información, que debe hacer un estudiante para resolverla. En el presente trabajo para calcular la demanda de la tarea se siguió el proceso establecido por El-Banna, (1987), de donde se ha tomado el siguiente ejemplo.

“¿Qué volumen de $\mathrm{HCl} 1.0 \mathrm{M}$ reaccionaría exactamente con 10.0 gramos de tiza?

1. La tiza es carbonato de calcio (recordar)

2. El Carbonato de calcio es $\mathrm{CaCO}_{3}$ (recordar)

3. La masa molecular del compuesto es $100 \mathrm{~g} \mathrm{~mol}^{-1}$ (recordar o calcular)

4. $10 \mathrm{~g}$ corresponden a $1 / 10$ de mol (deducir)

5. Escribir la ecuación para la reacción (recordar los productos y las fórmulas)

6. Balancear la ecuación (recordar habilidad)

7. Deducir la relación de moles

8. Por tanto, $1 / 10$ de mol de $\mathrm{CaCO}_{3}=1 / 5$ de mol de $\mathrm{HCl}$ (deducir)

9. $1 / 5$ de mol de $\mathrm{HCl}$ son $200 \mathrm{ml}$ del ácido $0.1 \mathrm{M}$ (deducir)"

Así, la demanda de esta tarea para un estudiante, cuya capacidad de procesar información no está tan desarrollada como la de su profesor, es 9, mientras que para un docente o un químico profesional seguramente es mucho menor, según el mencionado autor, puede ser tan pequeña como tres pasos complejos.

\section{Los resultados teóricos esperados}

\section{El concepto de valor de facilidad, $\mathrm{V}_{\mathrm{f}}$}

En el procedimiento seguido para analizar los resultados de la investigación, se ha definido un valor de facilidad, $V_{f}$, igual a: $\mathbf{V}_{\mathbf{f}}=\mathbf{n} / \mathbf{n t}$, donde $\mathrm{n}$ representa el número de estudiantes que respondieron correctamente una pregunta y nt es el número total de estudiantes que la debían responder; para analizar el desempeño de los grupos en las distintas pruebas, conformadas por preguntas de diferente demanda se grafica este valor contra la demanda de las distintas preguntas.

A manera de ilustración del procedimiento para calcular el valor de facilidad para una pregunta, supóngase que de 45 estudiantes que deberían responderla solamente lo hicieron correctamente 20 , entonces $V_{f}$ para dicha pregunta será igual a:

$$
\mathrm{V}_{\mathrm{f}}=20 / 45=0.44
$$

Por un procedimiento semejante al anterior, se determinaron los valores de facilidad para las distintas preguntas de las pruebas y del examen final del curso cuyos resultados se analizan en este artículo. De conformidad con el modelo de Johnstone, teniendo los valores de la capacidad mental provenientes de la aplicación del FIT y el DBT a uno o varios cursos, éstos se organizan en grupos de alumnos con la misma capacidad mental, $M=4, M=5, M=6, M=7$ y así sucesivamente; la construcción de una gráfica de $\mathrm{V}_{\mathrm{f}}$ contra la demanda de las preguntas para cada grupo de capacidad mental, conduce a la Figura 2, donde por simplicidad sólo se 
incluyen tres de los valores posibles para M. Esta figura corresponde a los resultados teóricos esperados para el desempeño ideal de los estudiantes en las pruebas elaboradas con preguntas de diferente demanda. (Johnstone e El-Banna, 1986).

Como se aprecia en la Figura 2, estudiantes cuya capacidad mental es 5 tendrían un desempeño alto en aquellas preguntas de demanda menor o igual a este valor, es decir los valores de facilidad para estas preguntas se acercarían a 1.00; sin embargo, este desempeño, expresado en términos de $\mathrm{V}_{\mathrm{f}}$ disminuiría rápidamente en la medida que la demanda de la pregunta es superior a 5 .

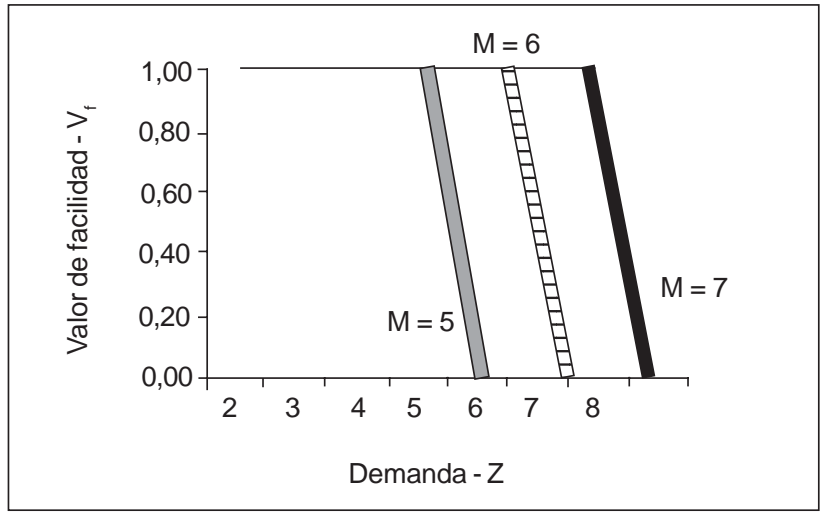

Figura 2. Resultados teóricos para un estudiante o un grupo de estudiantes con una capacidad mental dada.

De la misma manera, estudiantes con capacidades mentales 6 y 7 presentarían resultados semejantes, a menos que tuvieran o hubiesen desarrollado estrategias de procesamiento de información que les permitieran operar sobre la demanda de la pregunta para reducirla, en estos últimos casos, los valores de facilidad de las preguntas tenderían a ser nuevamente altos.

Así mismo, el poco éxito, bajos valores de facilidad para una pregunta en una prueba, podrían explicarse a partir de la carencia de estrategias de procesamiento de información, la aplicación de estrategias inadecuadas por parte del sujeto para resolverlas, o de una demanda excesiva de la pregunta para una capacidad mental dada de un estudiante. Conviene recordar sin embargo, que existen además otros factores diferentes a los anteriores que también influyen en el desempeño académico de los sujetos en un momento particular y que, para efectos de esta investigación, se relacionan básicamente con la preparación académica de los alumnos y la naturaleza propia de los temas objeto de estudio.

\section{Metodología}

El trabajo cuyos resultados se describen aquí, consistió en determinar para un curso de Química General de primer semestre, su capacidad de retensión y procesamiento de infor- 
Dificultades de aprendizaje en Química...

mación, o parámetro $\mathrm{M}$, mediante la aplicación de la prueba de los Dígitos Espaciados en su versión dígitos invertidos, y la prueba de las Figuras de Intersección, que como ya se mencionó son considerados instrumentos estandarizados y ampliamente aplicados en el área de investigación del aprendizaje en Química (Johnstone e El-Banna, 1986; Al-Naeme, 1989; El-Banna, 1987). Este curso pertenecía a la Facultad de Ingeniería de Alimentos y estaba integrado por 26 estudiantes de los cuales uno se retiró y 25 llegaron hasta el final; 17 de ellos eran hombres y 8 mujeres, 20 de ellos tenían una edad entre 16 y 20 años y 5 entre 21 y 25, el promedio de la edad en este curso era de 19 años.

A partir de las evaluaciones realizadas por el docente durante el semestre, se estructuraron tres pruebas, con diferente número de preguntas a las cuales se les estableció su demanda, así mismo, al cuestionario de examen final del curso se le estableció la demanda para cada una de las preguntas; las pruebas parciales dan información acerca del progreso de los estudiantes durante el desarrollo del semestre, mientras que el examen final proporciona información acerca de desempeño global de los estudiantes en el curso.

\section{El desempeño de los estudiantes en las pruebas de capacidad mental}

En la Tabla 1, se resumen los resultados correspondientes al desempeño de este grupo en el FIT y el DBT, como expresión de la capacidad mental.

Tabla 1. Distribución de los alumnos del curso según su capacidad mental, M

\begin{tabular}{cc}
\hline Capacidad Mental M & Frecuencia \\
\hline$M=8$ & 12 \\
$M=7$ & 4 \\
$M=6$ & 3 \\
$M=5$ & 3 \\
$M=4$ & 2 \\
$M=3$ & 1 \\
Total de estudiantes & 25 \\
\hline
\end{tabular}

De conformidad con los datos de la Tabla 1 se observa en este curso una tendencia hacia los valores más altos de la capacidad mental, en efecto, el $64.0 \%$ de los estudiantes se agrupan alrededor de valores de capacidad mental 7 y 8.

Par efectos del análisis entre la capacidad mental de los estudiante y las preguntas de diferente demanda, solamente se tuvieron en cuenta aquellos estudiantes cuyo valor de $\mathrm{M}$ es de cinco o más, por lo tanto, a partir de este criterio, de los 25 estudiantes referenciados en la tabla anterior, se excluyen 3; dos con $\mathrm{M}=4$ y uno con $\mathrm{M}=3$. De esta manera, el análisis que se continúa en seguida, corresponde a 22 alumnos.

Algunas de las razones para este bajo desempeño de estos tres estudiantes en los test pueden incluir una carencia de entendimiento de las instrucciones de la prueba o quizá poco interés en realizarla; es bien conocido que en muchos casos existe cierta tendencia, por parte de 
los estudiantes, a no prestar atención en el aula a todas aquellas actividades que conlleven pruebas que no sean tenidas en cuenta con fines de evaluación.

\section{Una visión global del desempeño académico de los estudiantes}

En razón de que el número de preguntas para cada una de las evaluaciones parciales y para el examen final, si bien fueron de diferente demanda, permaneció constante en número, 4 solamente, se consideró pertinente construir una prueba global, para este análisis, a partir de las preguntas incluidas en dichas evaluaciones, no hubo reaplicación de ninguna de ellas. Una prueba así construida, permite ampliar el rango de los valores de la demanda incluyendo preguntas de demanda baja, media y alta tomadas, al azar, de los parciales realizados durante el semestre. De esta manera, la prueba puede considerarse representativa de los contenidos desarrollados en el curso, de las evaluaciones realizadas durante el mismo y permite hacer un análisis más amplio del desempeño académico general de los estudiantes; una de tales combinaciones posibles se ilustra en la Tabla 2 y sus resultados se describen en seguida; nótese que cada una de las pruebas aporta por lo menos una pregunta, cuyos valores de facilidad se resumen en la Tabla 3.

Esta prueba, a manera de reconciliación integradora, se convierte en una forma de explicitar y discutir lo que sucedió a lo largo del curso en términos de rendimiento académico de los alumnos y representa una síntesis del análisis de los datos relacionados con este grupo de alumnos.

Los datos consignados en la Tabla 2 permiten establecer que la prueba global estuvo integrada por dos preguntas provenientes de la primera prueba, dos de la segunda, una de la tercera y dos del examen final.

A continuación se comentan los resultados obtenidos para cada grupo de capacidad mental en las preguntas seleccionadas, que abarcan demandas desde 2 hasta 8.

Tabla 2. Combinación de preguntas de diferente demanda para tener una visión global del rendimiento académico del curso.

\begin{tabular}{llllllllll}
\hline & Demanda Z & & 2 & 3 & 4 & 5 & 6 & 7 & 8 \\
\hline 1 & $x$ & & & & & & \\
2 & & $x$ & $x$ & & & & & \\
3 & & & & & $x$ & & \\
Exaeba No. & & & & $x$ & & $x$ & \\
\hline
\end{tabular}

Los datos resumidos en la Tabla 3 tienden a ser mayores para las capacidades mentales altas y para las preguntas de demanda más baja y viceversa, esto es, bajos para los valores bajos del parámetro M y para las preguntas de demanda alta, con ciertas excepciones; así mismo, ellos reflejan el trabajo de un grupo de estudiantes de rendimiento medio alto, como se pudo establecer a partir del concepto general emitido por el docente. 
Dificultades de aprendizaje en Química...

Tabla 3. Valores de facilidad para las preguntas de diferente demanda, en la prueba que representa la visión global del curso, según grupos de diferente capacidad mental, M.

\begin{tabular}{|c|c|c|c|c|c|c|c|c|}
\hline Grupos y M. & Frecuencia & 2 & 3 & 4 & 5 & 6 & 7 & 8 \\
\hline$M=8$ & 12 & 0,67 & 0,83 & 0,83 & 0,83 & 0,92 & 0,75 & 0,25 \\
\hline$M=7$ & 4 & 0,75 & 0,50 & 0,50 & 1,00 & 0,75 & 0,50 & 0,25 \\
\hline$M=6$ & 3 & 0,67 & 0,67 & 0,67 & 0,00 & 0,67 & 0,33 & 0,33 \\
\hline$M=5$ & 3 & 0,67 & 0,67 & 0,67 & 0,67 & 0,33 & 0,67 & 0,00 \\
\hline
\end{tabular}

\section{Grupo de capacidad mental 8}

En la Figura 3, se puede observar como los valores de facilidad para este grupo de capacidad mental, están por encima de 0,60; se acercan a los valores que predice la teoría cuya variación se muestra, en esta y en las siguientes figuras, en la trayectoria de líneas discontinuas de color rojo. Obsérvese sin embargo como el valor de facilidad cae rápidamente en la medida que la demanda de la pregunta tiende a 8 .

A pesar de todo, estrictamente siguiendo las predicaciones del modelo, solamente uno de los valores de facilidad, el correspondiente a la pregunta 6 , prácticamente coincide con él, lo cual por una parte, es significativo si se tiene en cuenta que la muestra es relativamente pequeña y por otra, reclama la continuidad de nuevas investigaciones cada vez mas con cantidades mayores de estudiantes.

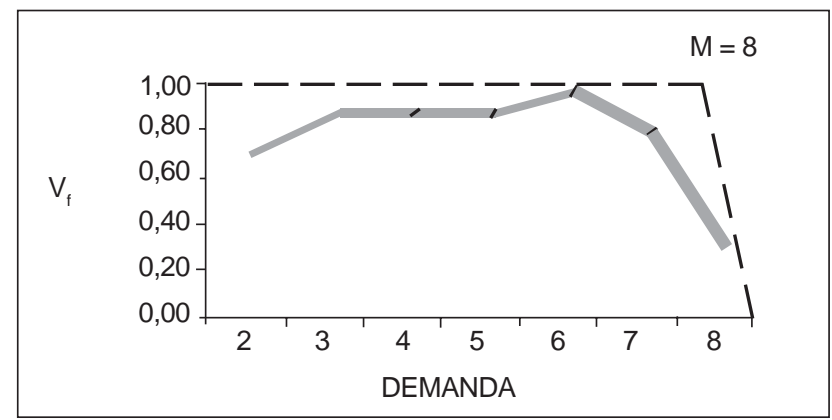

Figura 3. Desempeño de los estudiantes con capacidad mental 8 , en preguntas de diferente demanda, en la visión global del curso.

\section{Grupo de capacidad mental 7}

En comparación con los resultados presentados para el grupo de capacidad mental 8, los datos correspondientes a este grupo, Figura 4, presentan una variación menos regular; sin embargo, en términos generales, los valores de $V_{f}$ son relativamente altos y tienden a acercarse a la línea teórica hasta la pregunta de demanda 6 para luego descender en las vecindades del valor de la capacidad mental del grupo, 7. Una vez más, nótese que aparece también un dato que coincide exactamente con el valor teórico, 1.00. 
Cárdenas, F. A.

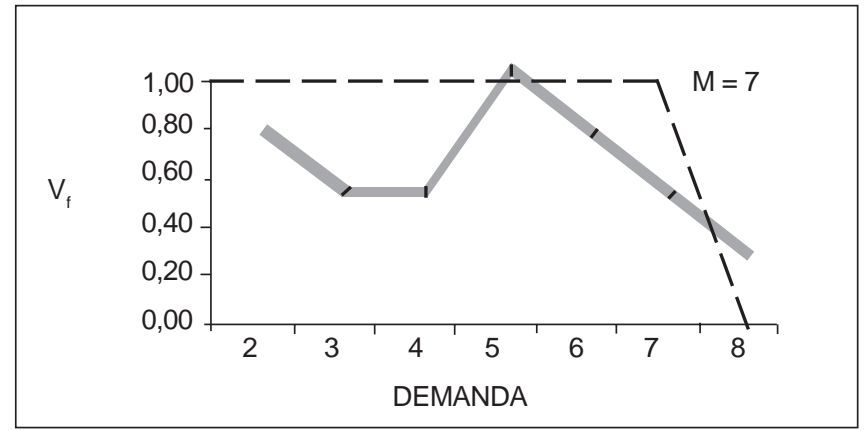

Figura 4. Desempeño de los estudiantes con capacidad mental 7 , en preguntas de diferente demanda, en la visión global del curso.

\section{Grupos de capacidad mental 6 y 5}

Con una disminución drástica en el valor de facilidad para la pregunta de demanda 5 , los valores correspondientes a las preguntas de demanda baja, 2, 3 y 4, son iguales, y tienden a acercarse al valor ideal para el grupo de capacidad mental 6, véase Figura 5. De la misma manera, para el grupo de capacidad mental 5, en la Figura 6 se puede observar que los valores de facilidad más altos son iguales entre sí y corresponden a las preguntas de demanda más baja. Sin embargo, en las dos graficas se observa un decrecimiento en el valor de facilidad una unidad antes, en el grupo de capacidad mental 6, Figura 5, y una unidad después en el grupo de capacidad mental 5, Figura 6. Por lo demás, ambos casos concuerdan con las expectativas teóricas en el sentido de mantener valores relativamente altos en las preguntas de demanda baja y valores relativamente bajos hacia la derecha, es decir, para las preguntas de demanda mayor.

En términos generales, esta visión global del curso explicita y resume las tendencias manifestadas parcialmente a lo largo del análisis de los resultados de las pruebas parciales y del examen final (Cárdenas, 2002) y conduce a pensar, que el rendimiento de este grupo de estudiantes es medio alto y esta asociado a los valores de la capacidad mental de sus integrantes, en concordancia con otras investigaciones (Cárdenas e Gonzalez, 2006)

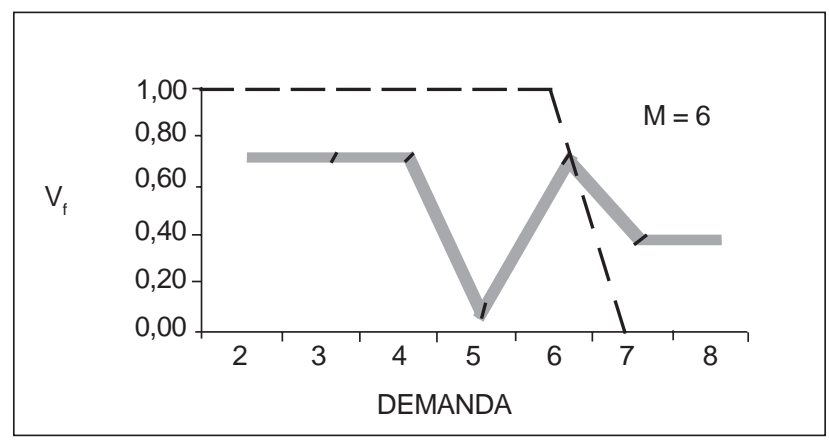

Figura 5. Desempeño de los estudiantes con capacidad mental 6 en preguntas de diferente demanda en la visión global del curso. 


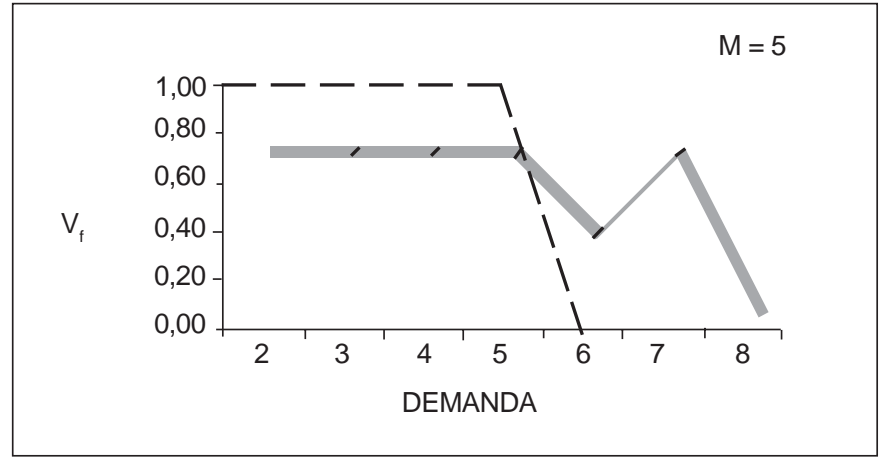

Figura 6. Desempeño de los estudiantes con capacidad mental 5, en preguntas de diferente demanda en la visión global del curso.

\section{El grado de dificultad de las unidades y sus respectivos subtemas}

De conformidad con las respuestas dadas por los estudiantes en el cuestionario de caracterización, en la Figura 7, se representan las unidades que conforman el programa de Química General y el porcentaje de estudiantes que las calificaron como difíciles. Nótese que las unidades más difíciles corresponden a las formulación y reacciones químicas, seguidas de la estequiometría y el equilibrio químico con igual grado de dificultad y luego las soluciones. En relación con estos resultados es preciso anotar que en su mayoría, los temas aquí identificados como difíciles coinciden con aquellos considerados como difíciles por estudiantes que han participado en otras investigaciones (Torres, 1996; Gonzalez, 2006), es el caso por ejemplo de las soluciones, y específicamente las soluciones sobresaturadas, la interpretación de las constantes de equilibrio en particular y las reacciones químicas, dentro de las cuales aparecen los procesos de balance por oxidación reducción.

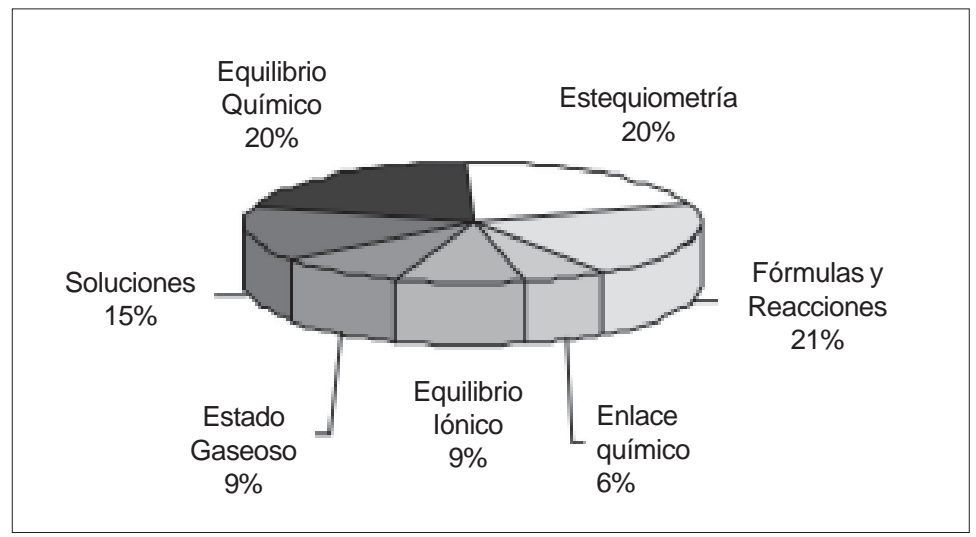

Figura 7. Unidades del programa de Química General y porcentaje de estudiantes que las consideraron como difíciles de aprender. 
Cuando se hace un recorrido analítico, en esta y en otras de nuestras investigaciones (Cárdenas, 2002; Peña, 2002), para comparar el valor de la demanda de algunas preguntas, usualmente incluidas en las evaluaciones parciales y en los exámenes finales y que alcanzan en muchos casos valores de 8,9 y hasta 10 (Cárdenas, 1999), con los temas de las unidades a las cuales pertenecen, se encuentra que las preguntas de mayor demanda corresponden a estequiometría, soluciones y el estado gaseoso, que son justamente algunas de las unidades consideradas por los estudiantes como las de mayor dificultad, véase Figura 7. En este sentido, un estudio actualmente en desarrollo, se orienta específicamente a estudiar la frecuencia de preguntas con altos valores de demanda y los temas de mayor dificultad presentes en evaluaciones de química. Se espera que sus resultados permitan una mejor interpretación de las relaciones entre estas dos variables y su incidencia en el desempeño de los estudiantes en las diferentes evaluaciones.

En términos de posibles explicaciones para el bajo rendimiento académico de estudiantes en la Química del primer semestre, en concordancia con los datos anteriores, es razonable pensar que sea bajo, si se tiene en cuenta que en las evaluaciones incluyendo la prueba del examen final, confluyen preguntas de demanda alta precisamente acerca de los temas más difíciles.

Desde el punto de vista pedagógico y en concordancia con los planteamientos teóricos es preciso tener en cuenta que se requiere de acciones intencionadas en el aula, e incluso fuera de ella, con los docentes, para que durante el tratamiento de estos temas y, sobre todo, en la preparación de las evaluaciones se tenga en cuenta la dificultad de cada uno de ellos y la elaboración de preguntas con una demanda adecuada a fin de que los resultados de la evaluación sean más válidos y confiables.

\section{Conclusiones}

El análisis de los resultados anteriores permite establecer que el grupo de estudiantes con los cuales se desarrolló esta investigación, en un alto porcentaje, presentó valores altos de capacidad mental, alrededor de 7 y 8 y que, su rendimiento académico en términos de desempeño frente a preguntas de diferente demanda se acercó a las predicciones teóricas, sin bien se presentaron algunas excepciones particularmente en los estudiantes de valores de capacidad mental baja.

En relación con los temas de mayor dificultad, en concordancia con los resultados de otras investigaciones, se pudo establecer que entre los señalados por este grupo de estudiantes como difíciles se encuentran la estequiometría, las soluciones y el estado gaseoso, en particular lo referente a la ecuación de estado.

Por otra parte, es de anotar, que por lo menos en parte, los bajos resultados de algunos de los estudiantes, sobre todo de aquellos que mostraron los menores niveles capacidad mental, se pueden explicar en términos de la confluencia de preguntas de alta demanda acerca de los temas que ellos consideran como de mayor dificultad. 
Dificultades de aprendizaje en Química...

\section{Referencias}

Al-NAEME, F. A. A study of some psychological factors affecting performance in chemistry at secondary and tertiary levels. M.Sc. Thesis. Center for science Education. Faculty of Science. University of Glasgow. 1989.

CÁRDENAS, S. F. A. Una aplicación de la teoría Neo-piagetiana a la enseñanza de la química. Informe final de investigación. Universidad Pedagógica nacional. Bogotá, Mayo de 1999.

Dificultades de Aprendizaje en Química: Caracterización y Búsqueda de Alternativas para Superarlas. Informe final de investigación. Oficina de Investigaciones. Universidad de La salle. Bogotá, Junio de 2002.

CÁRDENAS S. F. A.; GONZALEZ, M. F. Dificultades de Aprendizaje en Química: Caracterización y Búsqueda de Alternativas para Superarlas: Ampliación y continuación. Informe final de investigación. Oficina de Investigaciones. Universidad de La salle. Bogotá, Julio 26 de 2006.

CASE, R. Structures and Strictures, Some Functional Limitations on the Course of Cognitive Growth. Cognitive Psychology, v. 6, p. 544-573, 1974.

CASE, R.; GLOBERSON, T. Field Independence and Central Computing Space. Child Development, v. 45, p. 772-778, 1974.

CASE, R. Validation of a neo-Piagetian Capacity Construct. Journal of Experimental Child Psychology, v. 14, p. 287-302, 1972.

CASSELS, J. R. T.; JOHNSTONE, A. H. The Effect of Language on Students Performance on Multiple choice Tests in Chemistry. Journal of Chemical Education, v. 61, n. 7, p. 613615, 1984.

EL-BANNA, H. A. M. The Development of a Predictive Theory of Science Education Based Upon Information Processing Theory. Ph. D. Thesis. Center for Science Education. Faculty of Science. University of Glasgow, 1987.

GONZALEZ, M. F. Algunos factores que afectan el aprendizaje de la Química: la capacidad mental de los estudiantes y sus relaciones con las preguntas de diferente demanda. Tesis de maestría en docencia. Universidad de La Salle, 2006.

JOHNSTONE, H. A.; EL-BANNA, H. Capacities, Demands and Processes: a Predictive Model for Science Education. Education in Chemistry, v. 23, n. 3, p. 80-84, 1986. 
Cárdenas, F. A.

JOHNSTONE, H. A.; KELLET, M. C. Learning Difficulties in School Science. Towards a Working Hypothesis. European Journal of Science Education, v. 2, n. 2, p.175-181, 1980.

JOHNSTONE, H. A.; WHAM, B. J. The demands of practical work. Education in Chemistry, p. 71-73, 1982.

JOHNSTONE, H. A. New Stars for the Teacher to Steer by? Journal of Chemical Education, v. 65, n. 10, p. 847-849, 1984.

PASCUAL-LEONE, J. A Mathematical Model for the Transition Rule in Piaget's Developmental Stages. Acta Psicológica, v. 63, p. 301-345, 1970.

Manual for FIT: figural intersection test. Dpt. of Psychology. York University, 1967.

PEÑA, O. Algunos factores que afectan el rendimiento académico de los estudiantes en química. Tesis de maestría en Docencia. Universidad de La Salle. 2002.

SCARDAMALIA, M. Information Processing Capacity and the Problem of Horizontal Decalage: a demonstration using combinatorial reasoning tasks. Child development, v. 48, p. 2837, 1977.

TORRES, G. N. Una Aplicación del Pensamiento Neo-piagetiana en la Enseñanza de la Química. Tesis de Maestría en docencia de la Química. Universidad Pedagógica Nacional. 1996.

Artigo recebido em junho de 2006 e aceito em novembro de 2006 